\title{
INFINITESIMAL VARIATIONS OF SUBMANIFOLDS OF AN EVEN-DIMENSIONAL SPHERE
}

\author{
By KentaRo Yano and MasahiRo Kon
}

\section{$\S 0$. Introduction}

The present authors [3] have recently studied submanifolds of an evendimensional sphere and one of the present authors [2] studied infinitesimal variations of submanifolds of Riemannian manifolds.

The purpose of the present paper is to study infinitesimal variations of submanifolds of an even-dimensional sphere.

In $\S 1$, we state some of known results on the structure ([4], [5]) which an even-dimensional sphere admits and in $\S 2$ those on various kinds of submanifolds of an even-dimensional sphere (see [3]).

$\S 3$ is devoted to the study of infinitesimal variations of these submanifolds, $\S 4$ to the study of infinitesimal variations carrying an invariant submanifold of an even-dimensional sphere into an invariant one and $\S 5$ to the study of those carrying an anti-invariant submanifold of an even-dimensional sphere into an anti-invariant one.

\section{$\S 1$. Even-dimensional spheres}

Let $E^{2 m+2}$ be a $(2 m+2)$-dimensional Euclidean space with standard complex structure $F$. We denote a point $P$ in $E^{2 m+2}$ by its position vector $X$.

Let $M^{2 m}$ be a $2 m$-dimensional Riemannian manifold covered by a system of coordinate neighborhoods $\left\{U ; x^{h}\right\}$ and denote by $g_{j i}$ and $\nabla_{j}$ the metric tensor of $M^{2 m}$ and the operator of covariant differentiation with respect to $g_{j i}$ respectively. Here and in the sequel the indices $h, i, j, k, \cdots$ run over the range $\{1,2, \cdots, 2 m\}$.

We assume that $M^{2 m}$ is isometrically immersed in $E^{2 m+2}$ by the immersion $\imath: M^{2 m} \rightarrow E^{2 m+2}$, identify $i\left(M^{2 m}\right)$ with $M^{2 m}$ and represent the immersion by

$$
X=X(x) \text {. }
$$

Then the immersion being isometric, we have

$$
g_{j i}=X_{j} \cdot X_{\imath},
$$

where $X_{j}=\partial_{j} X\left(\partial_{j}=\partial / \partial x^{j}\right)$ and the dot indicates the inner product in $E^{2 m+2}$. The

Received July 9, 1977. 
inner product in $E^{2 m+2}$ satisfies $(F Y) \cdot(F Z)=Y \cdot Z$ for any vector fields $Y$ and $Z$.

We denote by $C$ and $D$ two matually orthogonal unit vectors normal to $M^{2 m}$. Then for the transforms $F X_{\imath}$ of $X_{\imath}$ by $F$, we have equations of the form

$$
F X_{i}=f_{\imath}{ }^{h} X_{h}+u_{i} C+v_{i} D,
$$

where $f_{\imath}{ }^{h}$ is a tensor field of type $(1,1)$ and $u_{\imath}, v_{i}$ are 1-forms of $M^{2 m}$.

For $F C$ and $F D$, we have equations of the form

$$
F C=-u^{h} X_{n}+\lambda D
$$

and

$$
F D=-v^{n} X_{h}-\lambda C
$$

respectively, where $u^{h}=u_{i} g^{i h}, v^{h}=v_{i} g^{i h}$ and $\lambda$ is a function, $g^{i h}$ being contravariant components of the metric tensor of $M^{2 m}$. we find

Applying $F$ to (1.3), (1.4) and (1.5) and using $F^{2}=-1$ and these equations,

$$
\begin{gathered}
f_{\imath}{ }^{t} f_{t}{ }^{h}=-\delta_{i}^{h}+u_{\imath} u^{h}+v_{i} v^{h}, \\
u_{t} f_{\imath}{ }^{t}=\lambda v_{i}, \quad v_{t} f_{\imath}{ }^{t}=-\lambda u_{\imath}, \\
f_{t}{ }^{h} u^{t}=-\lambda v^{h}, \quad f_{t}{ }^{h} v^{t}=\lambda u^{h}, \\
u_{t} u^{t}=1-\lambda^{2}, \quad u_{t} v^{t}=0, \quad v_{t} v^{t}=1-\lambda^{2} .
\end{gathered}
$$

Also, from $(1.3)$ and $\left(F X_{j}\right) \cdot\left(F X_{\imath}\right)=X_{j} \cdot X_{\imath}$, we find

$$
f_{j}^{t} f_{i}^{s} g_{t s}=g_{j i}-u_{j} u_{i}-v_{j} v_{i} .
$$

A structure defined by the set $\left(f_{\imath}{ }^{h}, g_{j i}, u_{\imath}, v_{i}, \lambda\right)$ satisfying $(1.6) \sim(1.10)$ is called an $(f, g, u, v, \lambda)$-structure [4], [5]. Thus a submanifold $M^{2 m}$ of codimension 2 of a $(2 m+2)$-dimensional Euclidean space admits an $(f, g, u, v, \lambda)$-structure depending on the choice of normals $C$ and $D$.

From (1.6) (1.10), we see that $f_{j i}=f_{j}{ }^{t} g_{t \imath}$ satisfies $f_{j i}=-f_{\imath j}$.

Equations of Gauss and those of Weingarten for $M^{2 m}$ are respectively

$$
\nabla, X_{i}=h_{j i} C+k_{j i} D
$$

and

$$
\nabla_{i} C=-h_{\imath}{ }^{h} X_{h}+\mu D, \quad \nabla_{i} D=-k_{\imath}{ }^{n} X_{h}-\mu C,
$$

where $h_{j i}$ and $k_{j i}$ are the second fundamental tensors of $M^{2 m}$ with respect to $C$ and $D$ respectively, $h_{2}{ }^{h}=h_{i t} g^{t h}, k_{\imath}{ }^{h}=k^{\prime}{ }_{i t} g^{t h}$ and $\mu$ is a function.

We now suppose that $M^{2 m}$ is a sphere $S^{2 m}(1)$ with radius 1 and with its center at the origin of $E^{2 m+2}$. Thus we can put $C=-X$ and choose $D$ as a unit vector field perpendicular to the hyperplane $E^{2 m+1}$ containing $S^{2 m}(1)$. Then 
$C$ is a concurrent vector field and $D$ is a parallel vector field. Thus from (1.12) we have $h_{\imath}{ }^{h}=\delta_{\imath}{ }^{h}, k_{\imath}{ }^{h}=0, \mu=0$ and consequently $h_{j i}=g_{j i}, k_{j i}=0$. Thus equations of Gauss and Weingarten reduce to

$$
\nabla_{\jmath} X_{i}=g_{j i} C, \quad \nabla_{j} C=-X_{\jmath}, \quad \nabla_{j} D=0 .
$$

Thus differentiating (1.3) covariantly along $S^{2 m}(1)$ and using (1.4) and (1.13), we find

$$
\begin{gathered}
\nabla_{\jmath} f_{\imath}{ }^{h}=-g_{j i} u^{h}+\delta_{j}^{h} u_{\imath}, \\
\nabla_{\jmath} u_{i}=f_{j i}, \\
\nabla_{j} v_{i}=\lambda g_{\jmath i} .
\end{gathered}
$$

Equation (1.15) shows that $u^{h}$ is a Killing vector field and (1.16) shows that $v^{h}$ is a conformal Killing vector field.

Differentiating (1.4) and (1.5) covariantly along $S^{2 m}(1)$ and using (1.3) and (1.13), we find

$$
\begin{aligned}
& \nabla_{i} u^{h}=f_{i}{ }^{h}, \\
& \nabla_{i} v^{h}=\lambda \delta_{i}^{h}, \\
& v_{i}=-\nabla_{i} \lambda,
\end{aligned}
$$

As we see from (1.9), the vanishing of $u^{h}$ or $v^{h}$ in a domain of $S^{2 m}(1)$ is equivalent to the fact that $\lambda= \pm 1$. But as (1.16) shows, the case in which $v^{h}=0$ and $\lambda= \pm 1$ never happens in a domain of $S^{2 m}(1)$. Thus, $u^{h} \neq 0, v^{h} \neq 0$ and $\lambda \neq \pm 1$ almost everywhere in $S^{2 m}(1)$.

\section{$\S 2$. Submanifolds of $S^{2 m}(1)$}

Let $M^{n}$ be an $n$-dimensional Riemannian manifold covered by a system of coordinate neighborhoods $\left\{V ; y^{a}\right\}$ and denote by $g_{c b}$ and $\nabla_{c}$ the fundamental metric tensor and the operator of covariant differentiation with respect to $g_{c b}$ respectively. Here and in the sequel, the indices $a, b, c, \cdots$ run over the range $\left\{1^{\prime}, 2^{\prime}, \cdots, n^{\prime}\right\}$.

We assume that $M^{n}$ is isometrically immersed in $S^{2 m}(1)$ by the immersion $i: M^{n} \rightarrow S^{2 m}(1)$ and identify $i\left(M^{n}\right)$ with $M^{n}$. We represent the immersion by

$$
x^{h}=x^{h}\left(y^{a}\right) \text {. }
$$

If we put

$$
B_{b}{ }^{h}=\partial_{b} x^{h} \quad\left(\partial_{b}=\partial / \partial y^{b}\right),
$$

then the immersion being isometric, we have 


$$
g_{c b}=B_{c}{ }^{3} B_{b}{ }^{2} g_{j i} .
$$

We choose $2 m-n$ mutually orthogonal unit vectors normal to $M^{n}$ and denote them by $C_{y}{ }^{h}$, where, here and in the sequel, the indices $x, y, z$ run over the range $\left\{(n+1)^{\prime}, \cdots,(2 m)^{\prime}\right\}$.

The transforms $f_{\imath}{ }^{n} B_{b}{ }^{2}$ of $B_{b}{ }^{2}$ by $f_{\imath}{ }^{h}$ are written in the form

$$
f_{\imath}{ }^{h} B_{b}{ }^{2}=f_{b}{ }^{a} B_{a}{ }^{h}-f_{b}{ }^{2} C_{z}{ }^{n}
$$

and the transforms $f_{\imath}{ }^{h} C_{y}{ }^{\imath}$ of $C_{y}{ }^{2}$ by $f_{\imath}{ }^{h}$ in the form

$$
f_{\imath}{ }^{h} C_{y}{ }^{\imath}=f_{y}{ }^{a} B_{a}{ }^{h}+f_{y}{ }^{x} C_{x}{ }^{h},
$$

$f_{b}{ }^{x}$ and $f_{y}{ }^{a}$ being related by

$$
f_{b y}=f_{y b},
$$

where $f_{b y}=f_{b}^{z} g_{z y}$ and $f_{y b}=f_{y}^{c} g_{c b}, g_{z y}$ being the metric tensor of the normal bundle of $M^{n}$.

When $f_{\imath}{ }^{n} B_{b}{ }^{2}$ are always tangent to $M^{n}$, that is, when $f_{b}{ }^{x}=0, M^{n}$ is said to be invariant. In order for $M^{n}$ to be invariant, it is necessary and sufficient that

$$
f_{b x}=f_{b}{ }^{y} g_{y x}=-f_{j i} B_{b}{ }^{j} C_{x}{ }^{2}=0 .
$$

When $f_{\imath}{ }^{n} B_{b}{ }^{2}$ are always normal to $M^{n}$, that is, $f_{b}{ }^{a}=0, M^{n}$ is said to be anti-invariant. In order for $M^{n}$ to be anti-invariant, it is necessary and sufficient that

$$
f_{b a}=f_{b}{ }^{c} g_{c a}=f_{j i} B_{b}{ }^{3} B_{a}{ }^{\imath}=0 .
$$

Equations of Gauss and those of Weingarten for $M^{n}$ are respectively written as

$$
\nabla_{c} B_{b}{ }^{h}=h_{c b}{ }^{x} C_{x}{ }^{h}
$$

and

$$
\nabla_{c} C_{y}{ }^{h}=-h_{c}{ }^{a} B_{a}{ }^{h},
$$

$\nabla_{c}$ denoting the van der Waerden-Bortolotti covariant differentiation along $M^{n}$, where $h_{c b}{ }^{x}$ are the second fundamental tensors of $M^{n}$ with respect to $C_{x}{ }^{h}, h_{c}{ }^{a}{ }_{y}$ $=h_{c b y} g^{b a}$ and $h_{c b y}=h_{c b}^{z} g_{z y}$.

(1) Submanifolds of $S^{2 m}(1)$ tangent to $u^{h}$

Suppose that a submanifold $M^{n}$ of $S^{2 m}(1)$ is tangent to $u^{h}$. Then we have equation of the form $B_{a}{ }^{h} u^{a}=u^{h}$, from which, differentiating covariantly along $M^{n}$ and using (1.17) and (2.4), we find

and consequently

$$
h_{c b}{ }^{x} u^{b} C_{x}{ }^{h}+B_{a}{ }^{n} \nabla_{c} u^{a}=f_{c}^{a} B_{a}{ }^{h}-f_{c}^{x} C_{x}{ }^{h},
$$




$$
\nabla_{c} u_{b}=f_{c b}, \quad h_{c b y} u^{b}=-f_{c y},
$$

$f_{c h}$ being skew-symmetric, we have

PRoposition 2.1 ([3]). Suppose that a submanifold $M^{n}$ of $S^{2 m}(1)$ is tangent to $u^{h}$, then $u^{h}$ is a Killing vector field of $M^{n}$. Moreover, in order for $M^{n}$ to be invariant in $S^{2 m}(1)$, it is necessary and sufficient that $u^{h}$ is in the principal direction with principal curvature zero.

PROPOSition 2.2 ([3]). In order for a submanıfold $M^{n}$ of $S^{2 m}(1)$ tangent to $u^{h}$ to be anti-invariant in $S^{2 m}(1)$, it is necassary and sufficient that $u^{h}$ is a parallet vector field of $M^{n}$.

(2) Submanifolds of $S^{2 m}(1)$ normal to $u^{h}$

Suppose that a submanifold $M^{n}$ of $S^{2 m}(1)$ is normal to $u^{h}$. Then we have $B_{b}{ }^{2} u_{i}=0$, from which, differentiating covariantly along $M^{n}$ and using (1.15) and (2.4), we find

$$
h_{c b}{ }^{x} C_{x}{ }^{2} u_{\imath}+B_{b}{ }^{2}\left(f_{c}{ }^{a} B_{a \imath}-f_{c}{ }^{x} C_{x \imath}\right)=0,
$$

where $B_{a i}=B_{a}{ }^{3} g_{j i}$ and $C_{x i}=C_{x}{ }^{j} g_{j i}$ and consequently

$$
h_{c b}{ }^{x} C_{x}{ }^{2} u_{i}+f_{c b}=0 .
$$

Thus, $h_{c b}{ }^{x} C_{x}{ }^{2} u_{2}$ being symmetric and $f_{c b}$ being skew-symmetric in $c$ and $b$, we have

$$
h_{c b}{ }^{x} C_{x}{ }^{2} u_{i}=0, \quad f_{c b}=0 .
$$

Thus we have

Proposition 2.3 ([3]). If a submanifold $M^{n}$ of $S^{2 m}(1)$ is normal to $u^{h}$, then $M^{n}$ is geodesic with respect to $u^{h}$ and is anti-invariant.

(3) Submanifolds of $S^{2 m}(1)$ tangent to $v^{h}$

Suppose that a submanifold $M^{n}$ of $S^{2 m}(1)$ is tangent to $v^{h}$. Then we have equation of the form $B_{a}{ }^{h} v^{a}=v^{h}$, from which, differentiating covariantly along $M^{n}$ and using (1.18), we have

$$
h_{c b}{ }^{x} v^{b} C_{x}{ }^{h}+B_{a}{ }^{h} \nabla_{c} v^{a}=\lambda B_{c}{ }^{h},
$$

and consequently

$$
\nabla_{c} v_{b}=\lambda g_{c b}, \quad h_{c b} v^{b}=0 .
$$

On the other hand, from (1.19), we have $v_{b}=-\nabla_{b} \lambda$ and consequently, from the first equation of (2.13), we have $\nabla_{c} \nabla_{b} \lambda=-\lambda g_{c b}$. Thus by a theorem of Obata [1], we have

Proposition 2.4 ([3]). If a complete connected submanfold $M^{n}$ of $S^{2 m}(1)$ is 
tangent to $v^{h}$, then $M^{n}$ is a sphere of radius 1 and $v^{h}$ is in the principal direction of $M^{n}$ with principal curvature zero.

(4) Submanifolds of $S^{2 m}(1)$ normal to $v^{h}$

Suppose that a submanifold $M^{n}$ of $S^{2 m}(1)$ is normal to $v^{h}$. Then we have $B_{b}{ }^{2} v_{i}=0$ or $B_{b}{ }^{i} \nabla_{i} \lambda=0$, which shows that $\lambda$ is constant along $M^{n}$. Differentiating $B_{b}{ }^{2} v_{i}=0$ covariantly along $M^{n}$ and using (1.16), we find

$$
h_{c b}{ }^{x} C_{x}{ }^{2} v_{i}+\lambda g_{c b}=0 \text {. }
$$

We also have $\nabla_{b} v^{h}=\lambda B_{b}{ }^{h}$ which shows that the normal $v^{h}$ to $M^{n}$ is parallel or concurrent according as $\lambda=0$ or $\lambda \neq 0$ along $M^{n}$. Thus we have

Proposition 2.5 ([3]). Suppose that a submanifold $M^{n}$ of $S^{2 m}(1)$ is normal to $v^{h}$. Then $\lambda=$ constant along $M^{n}$.

If $\lambda=0$ along $M^{n}$, then $M^{n}$ is on a great sphere of $S^{2 m}(1)$ and $\imath s$ geodesic with respect to $v^{h}$. Moreover $v^{h}$ is parallel along $M^{n}$.

If $\lambda \neq 0$ along $M^{n}$, then $M^{n}$ is on a small sphere of $S^{2 m}(1)$ and is umbilical with respect to $v^{h}$. Moreover $v^{h}$ is concurrent along $M^{n}$.

\section{§ 3. Infinitesimal variations of submanifolds of $\mathbf{S}^{2 m}(\mathbf{1})[2]$}

We now consider an infinitesimal variation

$$
\bar{x}^{h}=x^{h}+\xi^{h}(y) \varepsilon
$$

of a submanifold $M^{n}$ of $S^{2 m}(1)$, where $\xi^{n}$ is a vector field of $S^{2 m}(1)$ defined along $M^{n}$ and $\varepsilon$ is an infinitesimal. We then have $\bar{B}_{b}{ }^{h}=B_{b}{ }^{h}+\partial_{b} \xi^{h} \varepsilon$, where $\bar{B}_{b}{ }^{h}=\partial_{b} \bar{x}^{h}$ are $n$ linearly independent vectors tangent to the varied submanifold at varied point $\left(\bar{x}^{h}\right)$. We displace $\bar{B}_{b}{ }^{h}$ back parallelly from the varied point $\left(\bar{x}^{h}\right)$ to the original point $\left(x^{h}\right)$ and obtain $\tilde{B}_{b}{ }^{h}=B_{b}{ }^{h}+\left(\nabla_{b} \xi^{h}\right) \varepsilon$, neglecting terms of order higher than one with respect to $\varepsilon$. In the sequel, we neglect always terms of order higher than one with respect to $\varepsilon$. Thus putting $\delta B_{b}{ }^{h}=\widetilde{B}_{b}{ }^{h}-B_{b}{ }^{h}$, we obtain

$$
\delta B_{b}{ }^{h}=\left(\nabla_{b} \xi^{h}\right) \varepsilon \text {. }
$$

On the other hand, putting

$$
\xi^{h}=\xi^{a} B_{a}{ }^{h}+\xi^{x} C_{x}{ }^{h},
$$

we have

$$
\nabla_{b} \xi^{h}=\left(\nabla_{b} \xi^{a}-h_{b}{ }^{a}{ }_{x} \xi^{x}\right) B_{a}{ }^{h}+\left(\nabla_{b} \xi^{x}+h_{b a}{ }^{x} \xi^{a}\right) C_{x}{ }^{h} .
$$

When the tangent space at a point $\left(x^{h}\right)$ of the submanifold and that at the corresponding point $\left(\bar{x}^{h}\right)$ of the varied submanifold are parallel, the variation is said to be parallel. From (3.2) and (3.4) we see that in order for an infinitesimal variation to be parallel, it is necessary and sufficient that 


$$
\nabla_{b} \xi^{x}+h_{b a}^{x} \xi^{a}=0 .
$$

We denote by $\bar{C}_{y}{ }^{h} 2 m-n$ matually orthogonal unit normals to the varied submanifold and by $\tilde{C}_{y}{ }^{n}$ vectors obtained from $\bar{C}_{y}{ }^{n}$ by parallel displacement of $\bar{C}_{y}{ }^{h}$ from the varied point $\left(\bar{x}^{h}\right)$ back to the original point $\left(x^{h}\right)$. Putting $\delta C_{y}{ }^{h}$ $=\widetilde{C}_{y}{ }^{h}-C_{y}{ }^{h}$, we find [2]

$$
\bar{C}_{y}{ }^{h}=C_{y}{ }^{n}-\Gamma_{j i}^{h} \xi^{\jmath} C_{y}{ }^{2}+\delta C_{y}{ }^{n},
$$

where $\Gamma_{j i}^{h}$ are Christoffel symbols formed with $g_{j i}$. Assuming that $\delta C_{y}{ }^{h}$ are infinitesimals of order one with respect to $\varepsilon$ and putting

$$
\delta C_{y}{ }^{n}=\left(\eta_{y}{ }^{a} B_{a}{ }^{n}+\eta_{y}{ }^{x} C_{x}{ }^{h}\right) \varepsilon,
$$

we have

$$
\eta_{y}{ }^{a}=-\left(\nabla^{a} \xi_{y}+h_{b}{ }^{a} \xi^{b}\right),
$$

where $\xi_{y}=g_{y x} \xi^{x}$. Then $\eta_{y}^{x}$ appearing in (3.7) is a tensor field of the normal bundle of $M^{n}$ satisfying $\eta_{y x}+\eta_{x y}=0, \eta_{y x}$ being defined by $\eta_{y x}=\eta_{y}{ }^{z} g_{z x}$.

(1) Infinitesimal variations of submanifolds of $S^{2 m}(1)$ tangent to $u^{h}$

Suppose that a submanifold $M^{n}$ of $S^{2 m}(1)$ is tangent to $u^{h}$. Then we have equation of the form $B_{a}{ }^{h} u^{a}=u^{h}$. In order that the varied submanifold is also tangent to $u^{h}(\bar{x})$, it is necessary and sufficient that we have equation of the form $\bar{B}_{a}{ }^{h} \bar{u}^{a}=u^{h}(\bar{x})$, from which, putting $\bar{u}^{a}=u^{a}+\delta u^{a}$,

$$
\left(B_{b}{ }^{h}+\partial_{b} \xi^{h} \varepsilon\right)\left(u^{b}+\delta u^{b}\right)=u^{h}+\xi^{j} \partial_{j} u^{h} \varepsilon .
$$

Thus using (1.17), we find

$$
B_{b}{ }^{h} \delta u^{b}+\left[\left(\nabla_{b} \xi^{h}\right) u^{b}-f_{\imath}{ }^{h} \xi^{i}\right] \varepsilon=0 .
$$

On the other hand, using (2.4), (2.5) and (3.3), we find

$$
f_{\imath}{ }^{h} \xi^{i}=\left(f_{b}{ }^{a} \xi^{b}+f_{y}{ }^{a} \xi^{y}\right) B_{a}{ }^{h}+\left(-f_{b}{ }^{x} \xi^{b}+f_{y}{ }^{x} \xi^{y}\right) C_{x}{ }^{h} .
$$

Thus, substituting (3.4) and (3.10) into (3.9), we find

or using (2.11)

$$
\begin{aligned}
B_{b}{ }^{h} \delta u^{b} & +\left[\left(\nabla_{b} \xi^{a}-h_{b}{ }^{a}{ }_{x} \xi^{x}\right) u^{b}-\left(f_{b}{ }^{a} \xi^{b}+f_{y}{ }^{a} \xi^{y}\right)\right] B_{a}{ }^{h} \varepsilon \\
& +\left[\left(\nabla_{b} \xi^{x}+h_{b e}{ }^{x} \xi^{e}\right) u^{b}+\left(f_{b}{ }^{x} \xi^{b}-f_{y}{ }^{x} \xi^{y}\right)\right] C_{x}{ }^{h} \varepsilon=0,
\end{aligned}
$$

$$
\begin{aligned}
B_{b}{ }^{h} \delta u^{b} & +\left(u^{b} \nabla_{b} \xi^{a}-\xi^{b} \nabla_{b} u^{a}\right) B_{a}{ }^{h} \varepsilon \\
& +\left(u^{b} \nabla_{b} \xi^{x}-f_{y} \xi^{y}\right) C_{x}{ }^{h} \varepsilon=0 .
\end{aligned}
$$

from which

$$
\delta u^{a}=\left(\mathcal{L} u^{a}\right) \varepsilon,
$$


$\mathcal{L}$ denoting the Lie derivative with respect to $\xi^{a}$ and

$$
u^{b} \nabla_{b} \xi^{x}-f_{y}^{x} \xi^{y}=0
$$

Thus we have

PROPOSITION 3.1. In order for an infinitesimal variation (3.1) to carry a submanifold $M^{n}$ of $S^{2 m}(1)$ tangent to $u^{n}$ into a submanifold $\bar{M}^{n}$ also tangent to $u^{h}(\bar{x})$, it is necessary and sufficient that (3.12) holds, the variation of $u^{a}$ being given by (3.11).

Since $f_{y x}=-f_{x y}$, we have, from (3.12),

$$
u^{b} \nabla_{b}\left(\xi^{x} \xi_{x}\right)=0 .
$$

Thus we have

COROLlary. Suppose that an infinitesimal variation carries a submanifold $M^{n}$ of $S^{2 m}(1)$ tangent to $u^{h}$ into a submanifold $\bar{M}^{n}$ also tangent to $u^{h}(\bar{x})$. Then the length of the normal part of the variation vector is constant in the direction of $u^{h}$.

(2) Infintesimal variations of submanfolds of $S^{2 m}(1)$ normal to $u^{h}$

Suppose that a submanifold $M^{n}$ of $S^{2 m}(1)$ is normal to $u^{h}$. Then we have the equation $B_{b}{ }^{2} u_{i}=0$. In order that the varied submanifold is also normal to $u^{h}(\bar{x})$, it is necessary and sufficient that we have $\bar{B}_{b}{ }^{2} u_{i}(\bar{x})=0$, from which

$$
\left[B_{b}{ }^{2}+\left(\partial_{b} \xi^{i}\right) \varepsilon\right]\left[u_{i}+\xi^{j} \partial_{j} u_{i} \varepsilon\right]=0 .
$$

Thus, using (1.15), we find

$$
\left(\nabla_{b} \xi^{i}\right) u_{i}+f_{j i} \xi^{\jmath} B_{b}{ }^{2}=0
$$

Substituting (3.4) and (3.10) into this equation, we have

$$
\begin{aligned}
& {\left[\left(\nabla_{b} \xi^{a}-h_{b}{ }^{a}{ }_{x} \xi^{x}\right) B_{a}{ }^{2}+\left(\nabla_{b} \xi^{x}+h_{b e}{ }^{x} \xi^{e}\right) C_{x}{ }^{2}\right] u_{\imath}} \\
& +\left[\left(f_{c}{ }^{a} \xi^{c}+f_{y}{ }^{a} \xi^{y}\right) B_{a i}+\left(-f_{c}{ }^{x} \xi^{c}+f_{y}{ }^{x} \xi^{y}\right) C_{x \imath}\right] B_{b}{ }^{2}=0,
\end{aligned}
$$

from which

$$
\left(\nabla_{b} \xi^{x}+h_{b e} \xi^{e}\right) C_{x}{ }^{2} u_{i}+f_{c b} \xi^{c}+f_{y b} \xi^{y}=0 .
$$

But we know that in this case $h_{b e}{ }^{x} u_{x}=0$ and $f_{c b}=0$. Thus the above equation becomes

$$
\left(\nabla_{b} \xi^{x}\right) u_{x}+f_{x b} \xi^{x}=0
$$

Thus we have

PROPOSITION 3.2. In order for an infinitesimal variation (3.1) to carry a submanifold $M^{n}$ of $S^{2 m}(1)$ normal to $u^{h}$ into a submanifold normal to $u^{h}(\bar{x})$, it is 
necessary and sufficient that (3.13) holds.

(3) Infintesimal variations of subamanfolds of $S^{2 m}(1)$ tangent to $v^{h}$

Suppose that a submanifold $M^{n}$ of $S^{2 m}(1)$ is tangent to $v^{h}$. Then we have equation of the form $B_{a}{ }^{h} v^{a}=v^{h}$. In order that the varied submanifold is also tangent to $v^{h}(\bar{x})$, it is necessary and sufficient that we have equation of the form $\bar{B}_{a}{ }^{h} \bar{v}^{a}=v^{h}(\bar{x})$, from which, putting $\bar{v}^{a}=v^{a}+\delta v^{a}$,

$$
\left[B_{b}{ }^{h}+\partial_{b} \xi^{h} \varepsilon\right]\left[v^{b}+\delta v^{b}\right]=v^{h}+\left(\xi^{j} \partial_{j} v^{h}\right) \varepsilon .
$$

Thus, using (1.18), we have

$$
B_{a}^{h} \delta v^{a}+\left[\left(\nabla_{b} \xi^{h}\right) v^{b}-\lambda \xi^{h}\right] \varepsilon=0 .
$$

Thus, substituting (3.3) and (3.4) into this equation, we find

$$
\begin{aligned}
B_{a}{ }^{h} \delta v^{a} & +\left[\left(\nabla_{b} \xi^{a}-h_{b}{ }^{a}{ }_{x} \xi^{x}\right) v^{b}-\lambda \xi^{a}\right] B_{a}{ }^{h} \varepsilon \\
+ & {\left[\left(\nabla_{b} \xi^{x}+h_{b e}{ }^{x} \xi^{e}\right) v^{b}-\lambda \xi^{x}\right] C_{x}{ }^{h} \varepsilon=0, }
\end{aligned}
$$

from which, using (2.13),

$$
B_{a}{ }^{h} \delta v^{a}+\left(v^{b} \nabla_{b} \xi^{a}-\lambda \xi^{a}\right) B_{a}{ }^{h} \varepsilon+\left(v^{b} \nabla_{b} \xi^{x}-\lambda \xi^{x}\right) C_{x}{ }^{h} \varepsilon=0,
$$

and consequently, using $\lambda \xi^{a}=\left(\nabla_{b} v^{a}\right) \xi^{b}$,

$$
\delta v^{a}=\mathcal{L} v^{a} \varepsilon
$$

$\mathcal{L}$. denoting the Lie derivative with respect to $\xi^{a}$ and

$$
v^{b} \nabla_{b} \xi^{x}-\lambda \xi^{x}=0
$$

Thus we have

PROPOSITION 3.3. In order for an infinitesimal variation (3.1) to carry a submanifold $M^{n}$ of $S^{2 m}(1)$ tangent to $v^{h}$ into a submanifold tangent to $v^{h}(\bar{x})$, it is necessary and sufficient that (3.15) holds, the variation of $v^{a}$ being given by (3.14).

(4) Infintesimal variations of submanifolds of $S^{2 m}(1)$ normal to $v^{h}$

Suppose that a submanifold $M^{n}$ of $S^{2 m}(1)$ is normal to $v^{h}$. Then we have equation of the form $B_{b}{ }^{2} v_{i}=0$. In order that the varied submanifold is also normal to $v^{h}(\bar{x})$, it is necessary and sufficient that we have $\bar{B}_{b}^{2} v_{i}(\bar{x})=0$, from which

$$
\left[B_{b}{ }^{2}+\left(\partial_{b} \xi^{i}\right) \varepsilon\right]\left[v_{i}+\left(\xi^{j} \partial_{j} v_{i}\right) \varepsilon\right]=0 .
$$

Thus using (1.16), we have

$$
\left(\nabla_{b} \xi^{i}\right) v_{i}+\lambda B_{b}{ }^{2} \xi_{i}=0
$$

Therefore substituting (3.4) into this equation, we have 
or, using (2.14),

$$
\left(\nabla_{b} \xi^{x}+h_{b e}{ }^{x} \xi^{e}\right) v_{x}+\lambda \xi_{b}=0,
$$

$$
\left(\nabla_{b} \xi^{x}\right) v_{x}=0
$$

Proposition 3.4. In order for an infinitesimal variation (3.1) to carry a submanifold $M^{n}$ of $S^{2 m}(1)$ normal to $v^{h}$ into a submanifold normal to $v^{h}(\bar{x})$, it is necessary and sufficient that (3.16) holds.

From (3.5) and (3.16), we have

COROLlary. An infinitesimal normal parallel variation carres a submanifold $M^{n}$ of $S^{2 m}(1)$ normal to $v^{h}$ into a submanifold normal to $v^{h}(\bar{x})$.

\section{$\S 4$. Infinitesimal variations carrying an invariant sumbmanifold of $S^{2 m}(1)$ into an invariant one}

We assume that $M^{n}$ is an invariant submanifold of $S^{2 m}(1)$. We then have

$$
f_{\imath}{ }^{h} B_{b}{ }^{2}=f_{b}{ }^{a} B_{a}{ }^{h}, \quad f_{\imath}{ }^{h} C_{y}{ }^{2}=f_{y}{ }^{x} C_{x}{ }^{h} .
$$

Differentiating the first equation of (4.1) covariantly along $M^{n}$ and using (1.14), we find

$$
\begin{array}{r}
\left(-g_{j i} u^{h}+\delta_{j}^{h} u_{\imath}\right) B_{c}{ }^{j} B_{b}{ }^{2}+f_{\imath}{ }^{h} h_{c b}{ }^{x} C_{x}{ }^{\imath} \\
=\left(\nabla_{c} f_{b}{ }^{a}\right) B_{a}{ }^{h}+f_{b}{ }^{e} h_{c e^{x}} C_{x}{ }^{h},
\end{array}
$$

from which, putting $u^{h}=u^{a} B_{a}{ }^{h}+u^{x} C_{x}{ }^{h}$ and using (4.1),

$$
\begin{gathered}
-g_{c b}\left(u^{a} B_{a}{ }^{h}+u^{x} C_{x}{ }^{h}\right)+B_{c}{ }^{h} u_{b}+h_{c b}{ }^{y} f_{y}{ }^{x} C_{x}{ }^{h} \\
=\left(\nabla_{c} f_{b}{ }^{a}\right) B_{a}{ }^{n}+h_{c e}{ }^{x} f_{b}{ }^{e} C_{x}{ }^{h},
\end{gathered}
$$

and consequently, comparing the tangential and normal parts,

$$
\nabla_{c} f_{b}^{a}=-g_{c b} u^{a}+\delta_{c}^{a} u_{b}
$$

and

$$
h_{c b}^{y} f_{y}^{x}-h_{c e}{ }^{x} f_{b}^{e}=g_{c b} u^{x},
$$

from which, taking the skew-symmetric part,

$$
h_{c e}{ }^{x} f_{b}^{e}=h_{b e}{ }^{x} f_{c}^{e} .
$$

Differentiating the second equation of (4.1) covariantly along $M^{n}$ and using (1.14), we find

$$
\begin{aligned}
\left(-g_{j i} u^{h}+\delta_{j}^{h} u_{\imath}\right) B_{c}{ }^{j} C_{y}{ }^{2}-f_{\imath}{ }^{h} h_{c}{ }^{a}{ }_{y} B_{a}{ }^{\imath} \\
=\left(\nabla_{c} f_{y}{ }^{x}\right) C_{x}{ }^{h}-f_{y}{ }^{x} h_{c}{ }^{a}{ }_{x} B_{a}{ }^{h},
\end{aligned}
$$


from which, using (4.1),

$$
B_{c}{ }^{h} u_{y}-h_{c}{ }_{y} f_{e}{ }^{a} B_{a}{ }^{h}=\left(\nabla_{c} f_{y}{ }^{x}\right) C_{x}{ }^{h}-h_{c}{ }^{a}{ }_{x} f_{y}{ }^{x} B_{a}{ }^{h},
$$

and consequently

$$
h_{c}{ }^{e} f_{e}{ }^{a}-h_{c}{ }^{a}{ }_{x} f_{y}{ }^{x}=\delta_{c}^{a} u_{y},
$$

which is equivalent to (4.3) and

$$
\nabla_{c} f_{y}{ }^{x}=0 \text {. }
$$

We now consider an infinitesimal variation (3.1) and assume that it carries the invariant submanifold $M^{n}$ of $S^{2 m}(1)$ into an invariant submanifold. Then we have

$$
f_{\imath}{ }^{h}(x+\xi \varepsilon) \bar{B}_{b}{ }^{2}=\left(f_{b}{ }^{a}+\delta f_{b}{ }^{a}\right) \bar{B}_{a}{ }^{h},
$$

that is,

$$
\begin{aligned}
& \left(f_{\imath}{ }^{h}+\xi^{j} \partial_{\jmath} f_{\imath}{ }^{h} \varepsilon\right)\left(B_{b}{ }^{2}+\partial_{b} \xi^{2} \varepsilon\right) \\
& =\left(f_{b}{ }^{a}+\delta f_{b}{ }^{a}\right)\left(B_{a}{ }^{h}+\partial_{a} \xi^{h} \varepsilon\right),
\end{aligned}
$$

from which, using (1.14), we obtain

$$
\begin{gathered}
{\left[f_{\imath}{ }^{h}+\xi^{\jmath}\left(-\Gamma_{j t}^{h} f_{\imath}{ }^{t}+\Gamma_{j i}^{t} f_{t}{ }^{h}-g_{j i} u^{h}+\delta_{j}^{h} u_{\imath}\right) \varepsilon\right]\left(B_{b}{ }^{2}+\partial_{b} \xi^{2} \varepsilon\right)} \\
=\left(f_{b}{ }^{a}+\delta f_{b}{ }^{a}\right)\left(B_{a}{ }^{h}+\partial_{a} \xi^{h} \varepsilon\right),
\end{gathered}
$$

or

$$
\left(f_{\imath}{ }^{h} \nabla_{b} \xi^{2}-f_{b}{ }^{a} \nabla_{a} \xi^{h}-\xi_{b} u^{h}+u_{b} \xi^{h}\right)=\left(\delta f_{b}^{a}\right) B_{a}{ }^{h} .
$$

Thus substituting (3.4) into this equation, we have

$$
\begin{aligned}
& {\left[\left(\nabla_{b} \xi^{e}-h_{b}{ }^{e}{ }_{x} \xi^{x}\right) f_{e}{ }^{a} B_{a}{ }^{h}+\left(\nabla_{b} \xi^{y}+h_{b e}{ }^{y} \xi^{e}\right) f_{y}{ }^{x} C_{x}{ }^{h}\right.} \\
& \quad-f_{b}{ }^{e}\left(\nabla_{e} \xi^{a}-h_{e}{ }^{a}{ }_{x} \xi^{x}\right) B_{a}{ }^{h}-f_{b}{ }^{e}\left(\nabla_{e} \xi^{x}+h_{e d}{ }^{x} \xi^{d}\right) C_{x}{ }^{h} \\
& \left.\quad-\xi_{b}\left(u^{a} B_{a}{ }^{h}+u^{x} C_{x}{ }^{h}\right)+u_{b}\left(\xi^{a} B_{a}{ }^{h}+\xi^{x} C_{x}{ }^{h}\right)\right] \varepsilon=\left(\delta f_{b}{ }^{a}\right) B_{a}{ }^{h},
\end{aligned}
$$

where we have put $u^{h}=u^{a} B_{a}{ }^{h}+u^{x} C_{x}{ }^{h}$, from which

$$
\begin{aligned}
\delta f_{b}{ }^{a}= & {\left[\left(\nabla_{b} \xi^{e}-h_{b}{ }^{e}{ }_{x} \xi^{x}\right) f_{e}{ }^{a}-f_{b}{ }^{e}\left(\nabla_{e} \xi^{a}-h_{e}{ }^{a}{ }_{x} \xi^{x}\right)\right.} \\
& \left.-\xi_{b} u^{a}+u_{b} \xi^{a}\right] \varepsilon
\end{aligned}
$$

or, using (4.2) and (4.4),

$$
\delta f_{b}{ }^{a}=\left[\left(\mathcal{L} f_{b}{ }^{a}\right)-2 h_{b}{ }^{e} \xi^{x} f_{e}{ }^{a}\right] \varepsilon
$$

and 
or, using (4.3),

$$
\begin{gathered}
\left(\nabla_{b} \xi^{y}+h_{b e} \xi^{e}\right) f_{y} x-f_{b}^{e}\left(\nabla_{e} \xi^{x}+h_{e d}^{x} \xi^{d}\right) \\
-\xi_{b} u^{x}+u_{b} \xi^{x}=0,
\end{gathered}
$$

$$
\left(\nabla_{b} \xi^{y}\right) f_{y}^{x}-f_{b}^{e}\left(\nabla_{e} \xi^{x}\right)+u_{b} \xi^{x}=0
$$

Thus we have

Proposition 4.1. In order for an infinitesimal variation (3.1) to carry an invariant submanifold $M^{n}$ of $S^{2 m}(1)$ into an invariant one, it is necessary and sufficient that (4.8) holds, the variation of $f_{b}{ }^{a}$ being given by (4.7).

\section{§. Infinitesimal variations carrying an anti-invariant submanifold into an anti-invariant one}

We assume that $M^{n}$ is an anti-invariant submanifold of $S^{2 m}(1)$. We then have

$$
f_{\imath}{ }^{h} B_{b}{ }^{2}=-f_{b}{ }^{x} C_{x}{ }^{h}, \quad f_{\imath}{ }^{h} C_{y}{ }^{\imath}=f_{y}{ }^{a} B_{a}{ }^{h}+f_{y}{ }^{x} C_{x}{ }^{h} .
$$

Differentiating the first equation of (5.1) covariantly along $M^{n}$ and using (1.14), we find

$$
\begin{aligned}
\left(-g_{j i} u^{h}\right. & \left.+\delta_{j}^{h} u_{\imath}\right) B_{c}{ }^{j} B_{b}{ }^{\imath}+f_{\imath}{ }^{h} h_{c b}{ }^{x} C_{x}{ }^{\imath} \\
& =-\left(\nabla_{c} f_{b}{ }^{x}\right) C_{x}{ }^{h}+f_{b}{ }^{x} h_{c}{ }^{a}{ }_{x} B_{a}{ }^{h},
\end{aligned}
$$

from which, using (5.1),

$$
\begin{array}{r}
-g_{c b}\left(u^{a} B_{a}{ }^{h}+u^{x} C_{x}{ }^{h}\right)+B_{c}{ }^{h} u_{b}+h_{c b}{ }^{y}\left(f_{y}{ }^{a} B_{a}{ }^{h}+f_{y}{ }^{x} C_{x}{ }^{h}\right) \\
=-\left(\nabla_{c} f_{b}{ }^{x}\right) C_{x}{ }^{h}+h_{c}{ }^{a}{ }_{x} f_{b}{ }^{x} B_{a}{ }^{h},
\end{array}
$$

and consequently, comparing the tangential and normal parts

$$
h_{c b}{ }^{x} f_{x}{ }^{a}-h_{c}{ }^{a}{ }_{x} f_{b}{ }^{x}-g_{c b} u^{a}+\delta_{c}^{a} u_{b}=0
$$

and

$$
\nabla_{c} f_{b}^{x}=g_{c b} u^{x}-h_{c b} f_{y}^{x} .
$$

From (5.2), taking the skew-symmetric part, we have

$$
h_{c}{ }^{a}{ }_{x} f_{b} x-h_{b}{ }^{a}{ }_{x} f_{c}{ }^{x}=\delta_{c}^{a} u_{b}-\delta_{b}^{a} u_{c} .
$$

Differentiating the second equation of (5.1) covariantly along $M^{n}$ and using (1.14), we find

$$
\begin{aligned}
\left(-g_{j i} u^{h}\right. & \left.+\delta_{j}^{h} u_{\imath}\right) B_{c}{ }^{\jmath} C_{y}{ }^{2}-f_{\imath}{ }^{h} h_{c}{ }^{a}{ }_{y} B_{a}{ }^{2} \\
& =\left(\nabla_{c} f_{y}{ }^{a}\right) B_{a}{ }^{h}+f_{y}{ }^{e} h_{c e}{ }^{x} C_{x}{ }^{h}+\left(\nabla_{c} f_{y}{ }^{x}\right) C_{x}{ }^{h}-f_{y}{ }^{x} h_{c}{ }^{a}{ }_{x} B_{a}{ }^{h}
\end{aligned}
$$


from which, using (5.1),

$$
\begin{aligned}
& B_{c}{ }^{h} u_{y}+h_{c}{ }^{a}{ }_{y} f_{a}{ }^{x} C_{x}{ }^{h} \\
& \quad=\left(\nabla_{c} f_{y}{ }^{a}\right) B_{a}{ }^{h}+h_{c e}{ }^{x} f_{y}{ }^{e} C_{x}{ }^{h}+\left(\nabla_{c} f_{y}{ }^{x}\right) C_{x}{ }^{h}-h_{c}{ }^{a}{ }_{x} f_{y}{ }^{x} B_{a}{ }^{h} .
\end{aligned}
$$

Thus, comparing the tangential and normal parts, we have

$$
\nabla_{c} f_{y}{ }^{a}=\delta_{c}^{a} u_{y}+h_{c}{ }^{a}{ }_{x} f_{y}{ }^{x},
$$

which is equivalent to (5.3) and

$$
\nabla_{c} f_{y}{ }^{x}=h_{c}{ }^{a} f_{a} f^{x}-h_{c e}{ }^{x} f_{y}^{e} .
$$

We now consider an infinitesimal variation (3.1) and assume that it carries the anti-invariant submanifold $M^{n}$ into an anti-invariant submanifold. Then we have

$$
f_{\imath}{ }^{h}(x+\xi \varepsilon) \bar{B}_{b}{ }^{2}=-\left(f_{b}{ }^{x}+\delta f_{b}{ }^{x}\right) \bar{C}_{x}{ }^{h},
$$

that is, using (3.6) and (3.7),

$$
\begin{aligned}
& \left(f_{\imath}{ }^{h}+\xi^{j} \partial_{\jmath} f_{\imath}{ }^{h}\right)\left(B_{b}{ }^{2}+\partial_{b} \xi^{\imath} \varepsilon\right) \\
& \quad=-\left(f_{b}{ }^{x}+\delta f_{b}{ }^{x}\right)\left[C_{x}{ }^{h}-\Gamma_{j i}^{h} \xi^{\jmath} C_{x}{ }^{2} \varepsilon+\left(\eta_{x}{ }^{a} B_{a}{ }^{h}+\eta_{x}{ }^{y} C_{y}{ }^{h}\right) \varepsilon\right],
\end{aligned}
$$

from which, using (1.14), we obtain

$$
\begin{aligned}
& {\left[f_{\imath}{ }^{h}+\xi^{\jmath}\left(-\Gamma_{j t}^{h} f_{\imath}{ }^{t}+\Gamma_{j i}^{t} f_{t}{ }^{h}-g_{j i} u^{h}+\delta_{j}^{h} u_{\imath}\right) \varepsilon\right]\left[B_{b}{ }^{2}+\partial_{b} \xi^{\imath} \varepsilon\right]} \\
& \quad=-\left(f_{b}{ }^{y}+\delta f_{b}{ }^{y}\right)\left[C_{y}{ }^{h}-\Gamma_{j i}^{h} \xi^{\jmath} C_{y}{ }^{2} \varepsilon+\left(\eta_{y}{ }^{a} B_{a}{ }^{h}+\eta_{y}{ }^{x} C_{x}{ }^{h}\right) \varepsilon\right],
\end{aligned}
$$

that is,

$$
\begin{aligned}
& {\left[f_{\imath}{ }^{h}\left(\nabla_{b} \xi^{i}\right)-\xi_{b} u^{h}+u_{b} \xi^{h}\right]} \\
& \quad=-f_{b}{ }^{y}\left(\eta_{y}{ }^{a} B_{a}{ }^{h}+\eta_{y}{ }^{x} C_{x}{ }^{h}\right) \varepsilon-\left(\delta f_{b}{ }^{y}\right) C_{y}{ }^{h} .
\end{aligned}
$$

Thus, substituting (3.4) into the above equation, we find

$$
\begin{aligned}
{\left[-\left(\nabla_{b} \xi^{a}\right.\right.} & \left.-h_{b}{ }^{a}{ }_{y} \xi^{y}\right) f_{a}{ }^{x} C_{x}{ }^{h}+\left(\nabla_{b} \xi^{y}+h_{b e}{ }^{y} \xi^{e}\right)\left(f_{y}{ }^{a} B_{a}{ }^{h}+f_{y}{ }^{x} C_{x}{ }^{h}\right) \\
& \left.-\xi_{b}\left(u^{a} B_{a}{ }^{h}+u^{x} C_{x}{ }^{h}\right)+u_{b}\left(\xi^{a} B_{a}{ }^{h}+\xi^{x} C_{x}{ }^{h}\right)\right] \varepsilon \\
= & -f_{b}{ }^{y}\left(\eta_{y}{ }^{a} B_{a}{ }^{h}+\eta_{y}{ }^{x} C_{x}{ }^{h}\right) \varepsilon-\left(\delta f_{b}{ }^{y}\right) C_{y}{ }^{h},
\end{aligned}
$$

from which, comparing tangential and normal parts and using (3.8),

$$
\begin{aligned}
\left(\nabla_{b} \xi^{y}+h_{b e}{ }^{y} \xi^{e}\right) f_{y}{ }^{a}-\xi_{b} u^{a}+u_{b} \xi^{a} & \\
& =f_{b}{ }^{y}\left(\nabla^{a} \xi_{y}+h_{e}{ }^{a} \xi^{e}\right),
\end{aligned}
$$

which is, according to (5.2), equivalent to

$$
\left(\nabla_{b} \xi^{y}\right) f_{y}^{a}=f_{b}^{y} \nabla^{a} \xi_{y}
$$


and

$$
\begin{gathered}
\delta f_{b}{ }^{x}=\left[\left(\nabla_{b} \xi^{a}-h_{b}{ }^{a} \xi^{y}\right) f_{a}{ }^{x}-\left(\nabla_{b} \xi^{y}+h_{b e}{ }^{y} \xi^{e}\right) f_{y}{ }^{x}\right. \\
\left.+\xi_{b} u^{x}+u_{b} \xi^{x}-f_{b}{ }^{y} \eta_{y}{ }^{x}\right] \varepsilon .
\end{gathered}
$$

Thus we have

Proposition 5.1. In order for an infinitesimal variation (3.1) to carry an anti-invariant submanifold $M^{n}$ of $S^{2 m}(1)$ into an anti-invariant one, it is necessary and sufficient that (5.8) holds, the variation of $f_{b}{ }^{x}$ being given by (5.9).

From (3.5) and (5.7), we have

Proposition 5.2. An infinitesimal parallel variation carres an anti-invariant submanifold $M^{n}$ of $S^{2 m}(1)$ into an antl-invariant one if and only if $\xi^{a}$ is in the direction of $u^{a}$.

From (3.5) and (5.8), we have

PROPOSITION 5.3. An infinitesimal normal parallel variation carries always an anti-invariant submanifold $M^{n}$ of $S^{2 m}(1)$ into an anti-invariant one.

\title{
BIBLIOGRAPHY
}

[1] M. OвAтA, Riemannian manifolds admitting a solution of a certain system of differential equations, Proc. U.S.-Japan Seminar in Diff. Geom. Kyoto. Japan, 1965, 101-114.

[2] K. YANO, Infinitesimal variations of submanifolds, Kodai Math. J., 1 (1978), $30-40$.

[3] K. YANO AND M. KON, Submanifolds of an even-dimensional sphere, Geometriae Dedicata, 6 (1977), 131-139.

[4] K. Yano and M. OKumura, On $(f, g, u, v, \lambda)$-structures, Kōdai Math. Sem. Rep., 22 (1970), 401-423.

[5] K. YANO AND M. OKUMURA, Invariant submanifolds of a manifold with $(f, g$, $u, v, \lambda)$-structure, Kōdai Math. Sem. Rep., 24 (1972), 75-90.

\author{
Tokyo Institute of Technology \\ AND \\ SCIEnCE University of TOKyo
}

